\title{
Cost of opioid medication abuse with and without tampering in the USA
}

This article was published in the following Dove Press journal:

ClinicoEconomics and Outcomes Research

\section{Jeffrey Vietri' \\ Elizabeth T Masters ${ }^{2}$ \\ Alexandra I Barsdorf ${ }^{2}$ Jack Mardekian ${ }^{3}$}

'Health Outcomes Practice, Kantar Health, Horsham, PA, USA; ${ }^{2}$ Health Economics \& Outcomes Research, Pfizer Inc., New York, NY, USA; ${ }^{3}$ Statistical Research \& Data Science Center, Pfizer Inc., New York, NY, USA
Correspondence: Jeffrey Vietri Pfizer Inc., 500 Arcola Road, Collegeville, PA 19426, USA

Tel + I 4848657963

Fax + I 4848656422

Email jeffrey.vietri@pfizer.com
Objective: To provide per-patient estimates of the economic burden for opioid medication abuse with and without tampering.

Patients and methods: Adults in the US who participated in the 2010 and/or 2011 National Health and Wellness Survey were resurveyed to provide information on use and abuse of prescription opioids in the previous 3 months. Participants $(N=20,885)$ were categorized as those who abused and tampered $(n=107)$, abused without tampering $(n=118)$, those who reported using of opioids as prescribed $(n=981)$, and non-opioid controls $(n=19,679)$. Average wages from the Bureau of Labor Statistics and health care unit costs from the Truven MarketScan database were applied to self-reported work impairment (absenteeism, presenteeism, and overall work impairment) and health care resource utilization (health care provider visits, emergency room visits, hospitalizations, and drug rehabilitation) to estimate indirect and direct medical costs, respectively. Estimated mean costs for these groups were compared using analysis of variance, and generalized linear models were used to compare costs adjusted for confounders.

Results: Those who abused and tampered had significantly higher mean indirect (work impairment: $\$ 3,614$ vs $\$ 2,938, p<0.05$ ) and direct (health care use: $\$ 23,328$ vs $\$ 4,514, p<0.001$ ) costs over 3 months than those who abused without tampering. This included higher mean incremental costs for non-opioid-related medical visits ( $\$ 14,180$ vs $\$ 2,236, p<0.001)$, opioid-related medical visits $(\$ 8,790$ vs $\$ 2,223, p<0.001)$, and drug rehabilitation ( $\$ 358$ vs $\$ 55, p<0.001)$. Increased total direct costs were associated with tampering after adjusting for confounders $(p<0.001)$. Median incremental costs were also higher among those who tampered.

Conclusion: Tampering with prescription opioid medications is associated with significantly increased medical costs compared to abuse without tampering. Reducing tampering may provide net health care savings.

Keywords: direct costs, health care resource utilization, indirect costs, medication tampering, opioid abuse, work impairment, abuse deterrent

\section{Introduction}

Prescription opioid medications are highly effective pain relievers, with a longestablished role in the management of acute pain and moderate-to-severe chronic pain. ${ }^{1}$ Many individuals use opioids solely for their intended purpose. However, abuse of prescription opioid medications remains a widespread problem in the USA, with the abuse of prescription and illicit opioids officially declared a Nationwide Public Health Emergency. ${ }^{2}$ Non-medical use (NMU) of opioids - taking more than the prescribed dose of one's own medication, taking a medication prescribed for another individual, and/or misusing or abusing a medication - is estimated at 11.1 
million Americans each year. ${ }^{3}$ US societal costs attributable to prescription opioid NMU were estimated to be as high as $\$ 55.7$ billion in $2007 .{ }^{4}$ Chronic NMU of prescription pain medications increased by $74.6 \%$ from $2002-2003$ to 2009-2010..$^{5}$ Thousands of overdose deaths are attributed to prescription opioids each year, ${ }^{6,7}$ with mortality quadrupling from 1999 to $2010 .{ }^{8-10}$ Drug treatment admissions related to prescription opioids increased more than five-fold between 1998 and 2008, ${ }^{11}$ and emergency room (ER) visits related to prescription opioids increased by $183 \%$ from 2004 to $2011 .{ }^{12}$ These trends have spurred the development of guidelines with the aim to better control the prescription of opioids. ${ }^{13}$

One type of NMU is abuse, which we consider as use to obtain psychotropic effects, or more colloquially, "to get high". ${ }^{14}$ Individuals may tamper with medication to facilitate abuse. Major forms of tampering include ingestion (ie, chewing and swallowing), inhalation, injection, and smoking, with other methods being less prevalent. ${ }^{15}$ Vietri et al reported the prevalence of prescription opioid abuse during the previous 3 months among US adults to be $1.31 \%$, with approximately half $(0.67 \%)$ tampering during that time. ${ }^{15}$ Numerous routes of abuse were reported, though drugs were most commonly abused in their original form. In general, opioid abusers, and especially those who tamper with opioid medications, have much worse health outcomes than those who do not abuse or tamper with opioid medications. ${ }^{15}$ Unintended routes of abuse are associated with risks over and above those due to overconsumption of the medication, such as the increased risk of contracting HIV and other blood-borne illnesses in those who inject opioids with non-sterile needles. ${ }^{16}$

There are clear economic costs to consider, alongside the humanistic impact, including extensive workplace costs, health care costs, and criminal justice costs. In a 2014 review of the US economic burden of opioid abuse, mean annual excess health care costs for opioid abusers with private insurance ranged from $\$ 14,054$ to $\$ 20,546$ and from $\$ 5,874$ to $\$ 15,183$ for opioid abusers with Medicaid, when compared with control subjects. ${ }^{17}$ The US national estimate for perevent emergency department/hospital inpatient visits from a $20 \%$ random sample of opioid-related US hospital visits from 2006 to 2008 was $\$ 18,891 .^{18}$ In a study of Veterans Health Administration (VHA) patients abusing opioids from 2006 to 2009, adjusted overall annual health care costs for diagnosed opioid abuse patients were $\$ 28,882$, compared with $\$ 13,605$ for patients using opioids, but without an opioid abuse diagnosis. ${ }^{19}$

Little is known regarding the economic burden pertaining to particular routes of abuse, such as prescription opioid tampering. Vietri et al reported that tampering with opioid medications was associated with significantly greater impairment to work productivity and more frequent use of health care in US adults, compared with those who do not abuse or tamper with opioid medications. ${ }^{15}$ While these findings indicate the existence of an economic burden associated with prescription opioid tampering, the economic costs, including incremental costs, have not been presented in the literature. In this context, the objective of this study is to provide perpatient estimates of the economic burden of opioid medication abuse with and without tampering among US adults due to work impairment and health care resource use.

\section{Patients and methods}

\section{Data source}

The current study used the online survey previously described by Vietri et al. ${ }^{15}$ The design was a cross-sectional study administered among recent (in the past 3 months) users of prescription opioid medication in the US general population. These respondents were identified through screening of participants from the National Health and Wellness Survey (NHWS). The NHWS also provided data on work productivity and activity impairment (WPAI) and health care resource use from control individuals who indicated no use of opioids. The survey received approval from the Essex Institutional Review Board (Lebanon, NJ, USA). All respondents provided written informed consent prior to taking part; the consent form was administered online, with participants clicking a link to indicate their agreement to participate.

A total of 55,223 potential respondents from the 2010 and 2011 NHWS were invited to participate, and 25,864 respondents were screened for use of opioid medications in the previous 3 months. Specifically, they were then asked to reflect on any medications taken in the past 3 months, regardless of whether these medications were prescribed to them. The survey indicated that prescription opioid medications were the focus of the study, and provided a description of opioids and their use in pain. Respondents were then presented with a list of prescription opioid medications and asked to indicate which, if any, they had taken in the past 3 months. The list included both generic and brand names for each opioid followed by a note saying they may also be called by street names, and the street names for each drug were listed. A follow-up question asked respondents to indicate whether each opioid medication had been taken for pain and whether it had been taken "to get high." These were the only two options provided. Respondents who indicated that they used opioids for pain were subsequently asked if 
they had taken their medication according to their doctor's prescription. Those who indicated taking their medication in a way different from how it was prescribed were considered to have misused their prescription and were not included in the full survey. Otherwise, the respondent was considered to have medical use only.

A total of 1,242 reported either 1) abusing opioid medications (with or without use for pain) or 2) only medical use of opioids as directed, and completed the main opioid-specific questionnaire for this study. Consistent with the previous study using these data, those who provided unintelligible open-ended responses $(n=8)$ or reported tampering with the medication, but did not report abuse $(\mathrm{n}=28)$, on this questionnaire were excluded, resulting in a total of 1,206 respondents to the survey. Only responses to the screener questions were collected for respondents who did not self-report use or abuse of prescription opioids. NHWS data were retrieved from the database for all respondents who were screened. Disposition of respondents is presented in Figure 1.

\section{Measures}

\section{Opioid abuse}

Abuse was defined by self-reported use of a prescription opioid medication with the intention to get a "high" within the last 3 months.

\section{Frequency of abuse}

Respondents indicated how often in the previous 3 months they took each medication to get "high" using response options ranging from "less often than 1 day a month" to

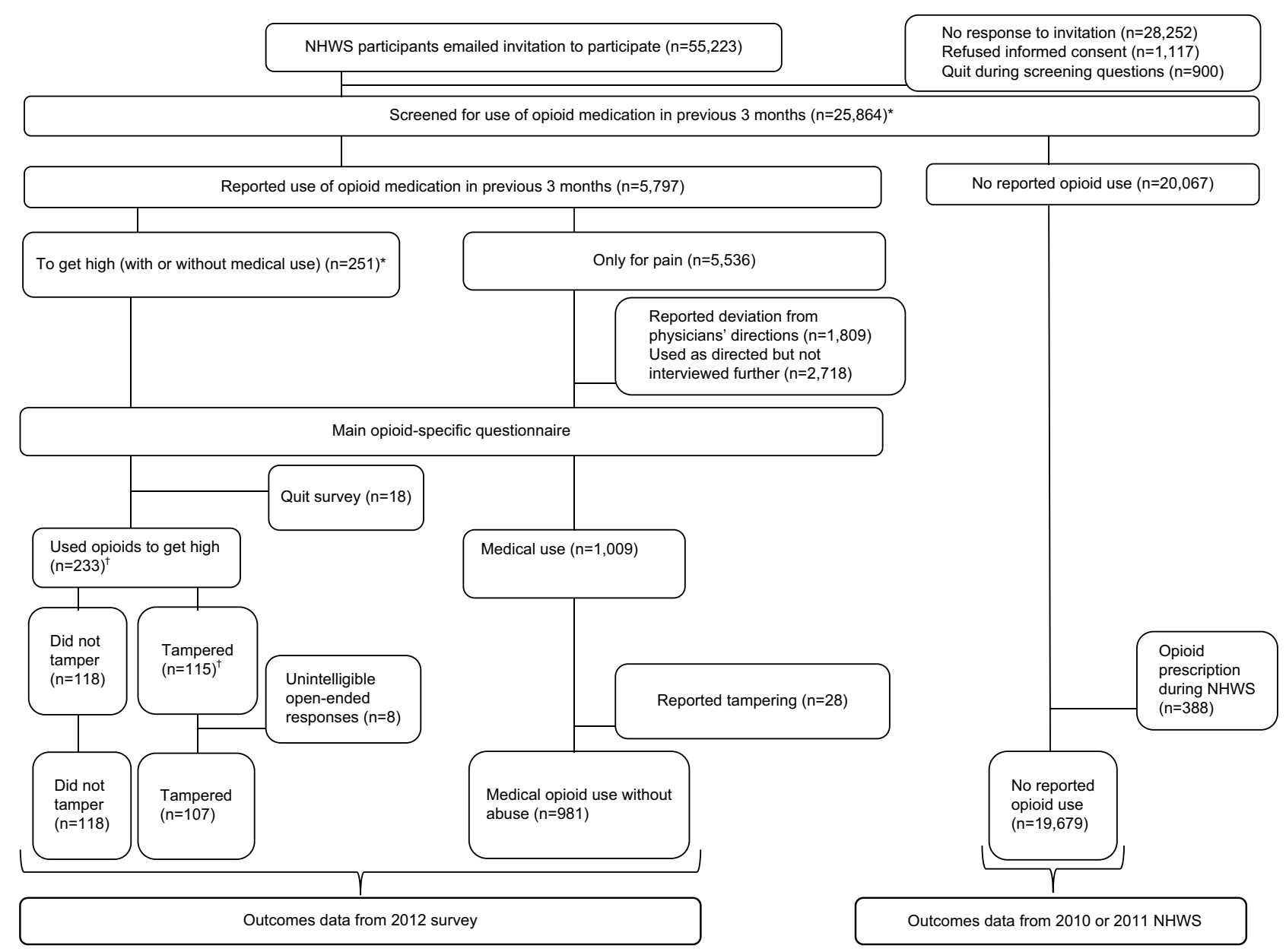

Figure I Disposition of the respondents.

Notes: Figures marked with an asterisk $\left(^{*}\right)$ were used to calculate prevalence of prescription opioid abuse, and figures with a dagger $\left({ }^{\dagger}\right)$ were used to calculate the prevalence of tampering in abuse in Vietri et al. ${ }^{15}$ Reproduced with permission from Vietri J, Joshi AV, Barsdorf Al, Mardekian J. Prescription opioid abuse and tampering in the United States: results of a self report survey. Pain Med. 2014;15(I2):2064-2074, by permission of Oxford University Press. ${ }^{15}$

Abbreviation: NHWS, National Health and Wellness Survey. 
"daily". A distinction between chronic and occasional abusers was made based on reported frequency of use pooled across all opioid drugs. Respondents who reported abusing opioid drugs $\leq 2$ times per week were considered occasional abusers. Respondents abusing $>2$ times per week were considered chronic abusers, under the rationale that abusing that frequently could not be limited to weekends or days off from a standard school or work week.

\section{Tampering}

For each medication, respondents indicated whether they took it as received (took it in the form I received it [e.g., swallowed pill, wore patch, placed suppository in rectum, injected it]), and/or whether they took it in a different form (took it in a different form [not as received - e.g., crushed, chewed, injected, infused into vein $\{$ intravenous/IV $\}$ - not an injection, snorted/ inhaled it, took through rectum, swallowed it with alcohol or any cough/cold medication containing alcohol]). Anyone who indicated that they took it in a form other than prescribed was considered to have tampered with the medication. Follow-up questions asked about which routes were used. Notably, while it could be argued that ingestion of an opioid with alcohol is not tampering, this was not relevant for the present analysis; no respondent was classified as tampering solely based on taking an opioid with alcohol, as all respondents who took an opioid with alcohol also used at least one other route of abuse listed above as tampering.

\section{Demographic and health characteristics}

The demographic characteristics of respondents included self-reported age, sex, marital status, employment status, health insurance, race, and annual household income, taken from the responses given in the 2011 or 2010 NHWS, linked by a panel ID number that preserved the anonymity of the respondents to the investigators. Information on the general health of the respondents was also taken from NHWS, including current cigarette smoking, alcohol use, exercise, body mass index (calculated from self-reported height and weight), and experience with and physician diagnosis of a variety of medical conditions, which were used to calculate the Charlson Comorbidity Index (CCI), ${ }^{20}$ as well as alcoholism, psychiatric illness (attention deficit hyperactivity disorder [ADHD], anxiety disorders, depression, bipolar disorder, or schizophrenia), and use of a prescription opioid for pain in the NHWS.

\section{Work productivity impairment}

These impairments were measured using two versions of the WPAI questionnaire. ${ }^{21}$ Only respondents who reported being employed full-time, part-time, or self-employed responded to the items measuring work impairment. Activity impairment, which was reported previously, ${ }^{15}$ was not used in the estimation of costs. All respondents completed the general health version of the WPAI (WPAI:GH) to assess impairment due to health overall, and those who reported using opioids in the past 7 days (the length of the recall period for the instrument) also completed the specific health problem version (WPAI:SHP), pertaining to their use (including abuse) of opioid medications. For those respondents who completed both versions, the higher of the two values for each of the three metrics (absenteeism, presenteeism, and overall work impairment) was used when making comparisons of health-related impairment. To avoid overestimation of overall work impairment, absenteeism and presenteeism were not combined across opioid-specific and general health versions.

\section{Indirect costs}

Indirect costs were based on productivity loss and were calculated using the 2011 US Department of Labor's Bureau of Labor Statistics average wages, stratified according to sex and age (18-24, 25-34, 35-44, 45-54, 55-64, and 65 or older). ${ }^{22}$ For each respondent, an hourly wage rate was estimated by dividing the median weekly income by 40 (the typical number of the hours worked in a week). Next, the number of hours missed in the last week because of one's health (absenteeism) and the number of hours missed in the last week because of health impairment while at work (presenteeism) were each multiplied by the hourly wage rates to arrive at total lost wages. These weekly costs were then multiplied by 50 (the typical number of work weeks in a year in the USA) to obtain annual estimates of the value of lost productivity. These costs were divided by four to estimate quarterly costs.

\section{Health care resource use}

Health care resource use was measured as the number of self-reported visits to health care providers, ER visits, and hospitalizations in the last 3 months. Respondents indicated the number of visits of each type specific to use (including abuse) of opioid medications and those unrelated to opioids. Respondents also indicated how many days during the past 3 months they participated in a variety of drug rehabilitation programs, including inpatient rehabilitation, detoxification programs, partial hospitalization programs, replacement therapy (methadone clinics), support groups, individual therapy, and group counseling. 


\section{Direct costs}

Direct health care costs were estimated from self-reported health care resource use. The 3-month reports of resource use were multiplied by the median cost of each service as reported in the 2011 Truven MarketScan database. The costs applied to opioid-related visits were based on the median cost of opioid abuse coded visits of each type (office, ER, hospital). The International Classification of Disease (ICD-9-CM) codes used were 304.7x (drug dependence involving opioids), 305.5x (opioid abuse), 965.00, 965.02, and 965.09 (poisoning by various opioids/opiates). The costs themselves are presented in Table 1. Unit costs for drug rehabilitation are taken from the Alcohol and Drug Services Study Cost Study (ADSSCS), ${ }^{23}$ which were inflated to 2011 dollars using the medical component of the consumer price index, likewise presented in Table 1. Information about the use of non-opioid medications was not included in the survey, and therefore pharmacy costs were not included in the calculations.

\section{Analysis}

Costs were compared across those who abused and tampered, those who abused without tampering, respondents reporting medical use only (as directed by their doctor), and the non-opioid using control group from the NHWS using chisquare tests for categorical variables and one-way analysis of variance (ANOVA) for continuous variables. In the case of significant $(p<0.05)$ results from the ANOVA, pairwise comparisons using Fisher's least significant difference test

Table I Per-service costs applied to each service in estimation of direct medical costs

\begin{tabular}{|c|c|c|c|}
\hline Services & $\begin{array}{l}\text { Opioid } \\
\text { related }\end{array}$ & Source & Amount (\$) \\
\hline \multicolumn{4}{|l|}{ Medical services } \\
\hline Outpatient visit & $\mathrm{N}$ & TMS & $\$ 108.01$ \\
\hline Outpatient visit & Y & TMS & $\$ 116.21$ \\
\hline ER & $\mathrm{N}$ & TMS & $\$ 552.86$ \\
\hline ER & Y & TMS & $\$ 633.11$ \\
\hline Hospitalization & $\mathrm{N}$ & TMS & $\$ 10,891.64$ \\
\hline Hospitalization & Y & TMS & $\$ 5,644.75$ \\
\hline \multicolumn{4}{|l|}{ Drug rehabilitation services } \\
\hline Group therapy & $Y$ & ADSSCS & $\$ 13$ \\
\hline Individual therapy & $Y$ & ADSSCS & $\$ 125$ \\
\hline Partial hospitalization program & Y & ADSSCS & $\$ 15$ \\
\hline Detoxification & Y & ADSSCS & $\$ 102$ \\
\hline Inpatient rehabilitation & Y & ADSSCS & $\$ 102$ \\
\hline Outpatient rehabilitation & $Y$ & ADSSCS & $\$ 15$ \\
\hline Methadone & $Y$ & ADSSCS & $\$ 17$ \\
\hline
\end{tabular}

Note: Values are 20II dollars.

Abbreviations: ADSSCS, Alcohol and Drug Services Study Cost Study; ER, emergency room; N, no; TMS, Truven MarketScan; $Y$, yes. were conducted to determine the significance of pairwise differences. No further adjustment for multiplicity was made. Median costs were also calculated, but were not compared using inferential statistics.

Generalized linear models were used to compare the medical costs of individuals who tampered with those who did not, controlling for potential confounders. All-cause direct and indirect costs were compared, as were opioid-specific direct costs. Models used a negative binomial probability distribution and a log-link function. Covariates included age, sex, annual household income $>\$ 50 \mathrm{k}$, non-Hispanic white race $(y / n)$, employed $(y / n), C C I$ scores, self-reported psychiatric illness ( $\mathrm{y} / \mathrm{n}$; includes any self-report of ADHD, depression, anxiety, or schizophrenia), obese BMI (y/n), consumes alcohol $(\mathrm{y} / \mathrm{n})$, current smoker $(\mathrm{y} / \mathrm{n})$, regular exercise $(\mathrm{y} / \mathrm{n})$, use of an opioid in NHWS, and possession of health insurance $(\mathrm{y} / \mathrm{n})$. Adjusted means (ie, regression-estimated mean costs equalizing the groups on all covariates) were calculated to facilitate interpretation.

\section{Results}

The sample included 107 respondents who abused and tampered, 118 who abused without tampering, 981 reporting medical use of opioids (as directed), and 19,679 respondents who did not report opioid abuse and had their NHWS data included as non-opioid controls. The sociodemographic characteristics of the opioid use groups are presented in Table 2. Most of the characteristics differed across the groups. Comparisons between those who abused and who did not abuse indicated that those who abused opioids were younger, less likely to be white, more likely to be male, smoke cigarettes, and drink alcohol than the rest of the sample (data not shown). Approximately $80 \%$ of those who tampered and abused prescription opioids abused them more than twice per week, approximately double the proportion of those who abused but did not tamper.

Indirect costs also varied according to use of prescription opioids, with higher costs among those abusing these medications (Table 3). Absenteeism costs were highest among tampering abusers; at $\$ 1,861 /$ quarter these costs were $~ 12$ times the amount of those who did not report any opioid use or abuse (ie, non-opioid controls) and $\sim 3$ times the mean cost of those with only medical use of opioids or those who abused, but did not tamper. Presenteeism costs were highest among non-tampering abusers at $\$ 2,276$; this estimate was 3 times the average costs incurred by non-opioid controls and $38 \%$ higher than the costs associated with the use of opioids for medical use only. Presenteeism costs between respondents 
Table 2 Demographic and health characteristics of the sample

\begin{tabular}{|c|c|c|c|c|c|c|c|c|c|}
\hline & \multicolumn{9}{|c|}{ Use of prescription opioids } \\
\hline & \multicolumn{6}{|c|}{ Current survey $(\mathrm{N}=\mathrm{I}, 206) *$} & \multirow{2}{*}{\multicolumn{2}{|c|}{$\begin{array}{l}\text { NHWS } \\
\text { Non-opioid } \\
\text { control }(n=19,679)\end{array}$}} & \multirow[t]{3}{*}{$p$-value } \\
\hline & \multicolumn{2}{|c|}{$\begin{array}{l}\text { Abuse with } \\
\text { tampering }(n=\mid 07)\end{array}$} & \multicolumn{2}{|c|}{$\begin{array}{l}\text { Abuse without } \\
\text { tampering }(n=\mid \text { I8) }\end{array}$} & \multicolumn{2}{|c|}{$\begin{array}{l}\text { Medical use only } \\
(n=981)\end{array}$} & & & \\
\hline & $\%$ & $\mathbf{n}$ & $\%$ & $\mathbf{n}$ & $\%$ & $\mathbf{n}$ & $\%$ & $\mathbf{n}$ & \\
\hline \multicolumn{10}{|l|}{ Demographics } \\
\hline Age, years (mean, SD) & 36.51 & 11.97 & 39.77 & 11.96 & 52.21 & $15.6 \mid$ & 50.42 & 15.83 & $<0.0001$ \\
\hline Female & 44.9 & 48 & 51.7 & 61 & 57.2 & 561 & 58.0 & 11415 & 0.0222 \\
\hline \multicolumn{10}{|l|}{ Race/ethnicity } \\
\hline White & $4 I .1$ & 44 & 58.5 & 69 & 80.2 & 787 & 80.1 & 15762 & $<0.0001$ \\
\hline Black & 16.8 & 18 & 11.9 & 14 & 9.2 & 90 & 8.1 & 1587 & \\
\hline Hispanic & 20.6 & 22 & 15.3 & 18 & 4.7 & 46 & 5.1 & 996 & \\
\hline Asian & 20.6 & 22 & 11.9 & 14 & 3.2 & 31 & 4.6 & 901 & \\
\hline Others & 0.9 & 1 & 2.5 & 3 & 2.8 & 27 & 2.2 & 433 & \\
\hline Married/living with partner & 55.1 & 59 & 57.6 & 68 & 59.6 & 585 & 62.2 & 12242 & 0.1225 \\
\hline \multicolumn{10}{|l|}{ Annual household income } \\
\hline$<\$ 25,000$ & 24.3 & 26 & 14.4 & 17 & 22.4 & 220 & 14.8 & 2906 & $<0.0001$ \\
\hline$\$ 25,000-\$ 49,999$ & 21.5 & 23 & 31.4 & 37 & 29.4 & 288 & 26.2 & 5154 & \\
\hline$\$ 50,000-\$ 74,999$ & 16.8 & 18 & 23.7 & 28 & 19.4 & 190 & 21.2 & 4179 & \\
\hline$\geq \$ 75,000$ & 33.6 & 36 & 28.0 & 33 & 20.4 & 200 & 26.8 & 5267 & \\
\hline Decline to answer & 3.7 & 4 & 2.5 & 3 & 8.5 & 83 & 11.0 & 2173 & \\
\hline Employed & 75.7 & 81 & 68.6 & 81 & 44.5 & 437 & 55.8 & 10982 & $<0.0001$ \\
\hline Health insurance & 70.1 & 75 & 68.6 & 81 & 87.5 & 858 & 85.6 & 16837 & $<0.000$ I \\
\hline \multicolumn{10}{|l|}{ Health characteristics } \\
\hline Currently smoke cigarettes & 27.1 & 29 & 39.8 & 47 & 20.5 & 201 & 13.1 & 2577 & $<0.0001$ \\
\hline Currently drink alcohol & 70.1 & 75 & 80.5 & 95 & 59.0 & 579 & 63.5 & 12502 & $<0.0001$ \\
\hline Currently exercise & 73.8 & 79 & 60.2 & 71 & 52.4 & 514 & 64.9 & 12762 & $<0.0001$ \\
\hline \multicolumn{10}{|l|}{ BMI categories } \\
\hline Underweight & 4.7 & 5 & 1.7 & 2 & 1.6 & 16 & 1.9 & 379 & $<0.0001$ \\
\hline Normal & 46.7 & 50 & 40.7 & 48 & 26.8 & 263 & 31.9 & 6274 & \\
\hline Overweight & 28.0 & 30 & 26.3 & 31 & 27.5 & 270 & 32.2 & 6331 & \\
\hline Obese & 18.7 & 20 & 30.5 & 36 & 42.3 & 415 & 31.8 & 6252 & \\
\hline Decline to answer & 1.9 & 2 & 0.8 & $\mathrm{I}$ & 1.7 & 17 & 2.3 & 443 & \\
\hline $\mathrm{CCl}($ mean, $\mathrm{SD})$ & 0.40 & 1.11 & 0.28 & 0.77 & 0.76 & 1.33 & 0.34 & 0.85 & $<0.0001$ \\
\hline Abuse $>2$ times/week & 80.4 & 86 & 41.5 & 49 & 0.0 & 0 & 0.0 & 0 & $<0.0001$ \\
\hline
\end{tabular}

Table 3 Estimated 3-month indirect costs by use of prescription opioids

\begin{tabular}{|c|c|c|c|c|c|c|c|c|c|c|c|c|}
\hline & \multicolumn{12}{|c|}{ Use of prescription opioids } \\
\hline & \multicolumn{9}{|c|}{ Current survey $(\mathrm{N}=\mathrm{I}, 206)^{*}$} & \multirow{2}{*}{\multicolumn{3}{|c|}{$\begin{array}{l}\text { NHWS } \\
\text { Non-opioid control } \\
(n=10,922)\end{array}$}} \\
\hline & \multicolumn{3}{|c|}{$\begin{array}{l}\text { Abuse with tampering } \\
(n=8 I)\end{array}$} & \multicolumn{3}{|c|}{$\begin{array}{l}\text { Abuse without tampering } \\
(n=8 I)\end{array}$} & \multicolumn{3}{|c|}{$\begin{array}{l}\text { Medical use only } \\
(n=433)\end{array}$} & & & \\
\hline & Mean & SD & Median & Mean & SD & Median & Mean & SD & Median & Mean & SD & Median \\
\hline Absenteeism & $\$ 1,86 I_{a}$ & $\$ 3,021$ & $\$ 672$ & $\$ 675_{b}$ & $\$ 1,853$ & $\$ 0$ & $\$ 518_{b}$ & $\$ 1,996$ & $\$ 0$ & $\$ 155_{c}$ & $\$ 974$ & $\$ 0$ \\
\hline Presenteeism & $\$ 1,824_{a, b}$ & $\$ 2,477$ & $\$ 947$ & $\$ 2,276_{a}$ & $\$ 3,252$ & $\$ 918$ & $\$ 1,645_{b}$ & $\$ 2,445$ & $\$ 513$ & $\$ 754_{c}$ & $\$ 1,676$ & $\$ 0$ \\
\hline Total & $\$ 3,614 a$ & $\$ 4,072$ & $\$ 2,133$ & $\$ 2,938_{b}$ & $\$ 3,729$ & $\$ 1,479$ & $\$ 2,166_{c}$ & $\$ 3,182$ & $\$ 890$ & $\$ 910_{d}$ & $\$ 2,029$ & $\$ 0$ \\
\hline
\end{tabular}

Notes: Sample size is reduced as analysis restricted to employed respondents not on vacation the week prior to the survey; means in the same row that do not share the same subscript letter $(a-d)$ are different at $p<0.05$ using the Fisher's LSD test. *Thirty-six of the I,242 respondents were excluded for unintelligible open-ended responses $(n=8)$ or tampered with the medication but did not report abuse $(n=28)$.

Abbreviations: LSD, least significant difference; NHWS, National Health and Wellness Survey. 
who abused did not differ according to tampering. When absenteeism and presenteeism costs were combined, these total costs were significantly higher with tampering abuse $\$ 3,614$ than non-tampering abuse, which were the next most expensive group. Median costs were generally consistent with the pattern of means. Notably, those who tampered were the only group with a median absenteeism cost above zero, and median indirect costs were $44 \%$ higher among those who abused and tampered relative to those who abused but did not tamper.

Direct costs were elevated in those who abused and tampered relative to all other groups for all of the comparisons, including physician visit costs, ER costs, hospitalizations, and drug rehabilitation ( $p<0.05$; Table 4$)$. Mean direct costs due to non-opioid-related health care resource use were $\$ 14,180, \sim 6$ times that of the next most expensive group (non-tampering abuse, \$2,236), for an incremental cost of $\$ 11,944$. The difference in costs was also significant for each type of service individually. Mean direct costs for opioid-related utilization were likewise elevated in those who abused and tampered, estimated at $\$ 8,790, \sim 4$ times the cost of those who abused but did not tamper $(\$ 2,223, p<0.05)$, for an incremental cost of $\$ 6,567$. When considered as total costs, those who abused and tampered incurred an estimated $\$ 23,328$ in direct medical expenses during the 3-month period, while non-tampering abusers incurred $\$ 4,514$, and non-abusing opioid users incurred $\$ 3,248$ (for all, $p<0.05$ ), for an unadjusted incremental cost of $\$ 18,814$ for abuse with tampering relative to abuse without tampering. All of these costs were greatly elevated relative to the $\$ 495$ estimated for the control group (for all, $p<0.05)$. Furthermore, the pattern was not driven wholly by outliers. Even after excluding any extreme responses, the group who tampered and abused was the only one to have non-zero costs for opioid-related care and drug rehabilitation, and these respondents also had the highest median costs for non-opioid-related care.

Adjusted indirect costs were not significantly higher among those who abused and tampered with the medication $(\$ 3,361)$ than among those who abused without tampering $(\$ 2,566 ; p=0.17)$. As in the unadjusted comparisons, the regression modeling indicated higher direct costs associated with tampering (Figure 2). All-cause direct costs were more than 6 times as high among those who tampered as those who abused but did not tamper $(\$ 20,289$ for those who tampered vs $\$ 2,971$ for those who did not, $p<0.001$; Figure 2). Likewise, the adjusted opioid-specific direct costs were almost 5 times as high among those who tampered relative to those who did not $(\$ 6,887$ vs $\$ 1,454$, $p<0.001)$.

Table 4 Estimated 3-month direct medical costs by use of prescription opioids

\begin{tabular}{|c|c|c|c|c|c|c|c|c|c|c|c|c|}
\hline & \multicolumn{12}{|c|}{ Use of prescription opioids } \\
\hline & \multicolumn{9}{|c|}{ Current survey $(N=I, 206)^{*}$} & \multirow{2}{*}{\multicolumn{3}{|c|}{$\begin{array}{l}\text { NHWS } \\
\text { Non-opioid control } \\
(n=19,679)\end{array}$}} \\
\hline & \multicolumn{3}{|c|}{$\begin{array}{l}\text { Abuse with tampering } \\
(n=107)\end{array}$} & \multicolumn{3}{|c|}{$\begin{array}{l}\text { Abuse without tampering } \\
(n=I \mid 8)\end{array}$} & \multicolumn{3}{|c|}{$\begin{array}{l}\text { Medical use only } \\
(n=98 I)\end{array}$} & & & \\
\hline & Mean & SD & Median & Mean & SD & Median & Mean & SD & Median & Mean & SD & Median \\
\hline \multicolumn{13}{|l|}{ Non-opioid related } \\
\hline Physician & $\$ 545_{a}$ & $\$ 891$ & $\$ 108$ & $\$ 217_{b}$ & $\$ 396$ & $\$ 108$ & $\$ 258_{b}$ & $\$ 357$ & $\$ 108$ & & & \\
\hline $\mathrm{ER}$ & $\$ 708_{a}^{a}$ & $\$ 1,164$ & $\$ 0$ & $\$ 173_{b}$ & $\$ 394$ & $\$ 0$ & $\$ 103_{b}$ & $\$ 311$ & $\$ 0$ & & & \\
\hline Hospitalization & $\$ 12,927$ & $\$ 31,244$ & $\$ 0$ & $\$ 1,846_{b}$ & $\$ 5,924$ & $\$ 0$ & $\$ 1,765_{b}$ & $\$ 5,580$ & $\$ 0$ & & & \\
\hline Total & $\$ 14,180_{a}$ & $\$ 32,245$ & $\$ 553$ & $\$ 2,236_{b}$ & $\$ 6,433$ & $\$ 108$ & $\$ 2,126_{b}$ & $\$ 5,837$ & $\$ 216$ & & & \\
\hline \multicolumn{13}{|l|}{ Opioid-related } \\
\hline Physician & $\$ 693_{a}$ & $\$ 1,084$ & $\$ 232$ & $\$ 163_{b}$ & $\$ 502$ & $\$ 0$ & $\$ 62_{c}$ & $\$ 129$ & $\$ 0$ & & & \\
\hline ER & $\$ 870_{a}$ & $\$ 1,346$ & $\$ 633$ & $\$ 290_{b}$ & $\$ 2,|2|$ & $\$ 0$ & $\$ 6_{c}$ & $\$ 86$ & $\$ 0$ & & & \\
\hline Hospitalization & $\$ 7,227_{a}$ & $\$ 11,881$ & $\$ 0$ & $\$ 1,770_{b}$ & $\$ 4,022$ & $\$ 0$ & $\$ 1,047_{b}$ & $\$ 3,176$ & $\$ 0$ & & & \\
\hline Total & $\$ 8,790_{a}$ & $\$ 13,546$ & $\$ 1,679$ & $\$ 2,223_{b}$ & $\$ 4,813$ & $\$ 0$ & $\$ 1,116_{c}$ & $\$ 3,196$ & $\$ 0$ & & & \\
\hline \multicolumn{13}{|c|}{ Overall (non-opioid related + opioid related) } \\
\hline Physician & $\$ 1,238_{a}$ & $\$ 1,904$ & $\$ 448$ & $\$ 380_{b}$ & $\$ 790$ & $\$ 116$ & $\$ 320_{c}$ & $\$ 390$ & $\$ 216$ & $\$ 170_{d}$ & $\$ 254$ & $\$ 108$ \\
\hline ER & $\$ 1,578_{a}$ & $\$ 2,339$ & $\$ 633$ & $\$ 463_{b}$ & $\$ 2,166$ & $\$ 0$ & $\$ 109_{c}$ & $\$ 332$ & $\$ 0$ & $\$ 27_{d}$ & $\$ 155$ & $\$ 0$ \\
\hline Hospitalization & $\$ 20,155$ & $\$ 38,602$ & $\$ 0$ & $\$ 3,616_{b}$ & $\$ 9,|4|$ & $\$ 0$ & $\$ 2,813_{c}$ & $\$ 7,849$ & $\$ 0$ & $\$ 299_{d}$ & $\$ 1,860$ & $\$ 0$ \\
\hline Drug rehabilitation & $\$ 358_{a}$ & $\$ 578$ & $\$ 49$ & $\$ 55_{b}$ & $\$ 204$ & $\$ 0$ & $\$ 7_{c}$ & $\$ 126$ & $\$ 0$ & $\$ 0_{d}$ & $\$ 0$ & $\$ 0$ \\
\hline Overall total & $\$ 23,328_{a}$ & $\$ 41,672$ & $\$ 4,019$ & $\$ 4,5 \mid 4_{b}$ & $\$ 10,434$ & $\$ 216$ & $\$ 3,248_{c}$ & $\$ 8,215$ & $\$ 224$ & $\$ 495_{d}$ & $\$ 1,993$ & $\$ 108$ \\
\hline
\end{tabular}

Notes: Means in the same row that do not share the same subscript letter (a-d) are different at $p<0.05$ using the Fisher's LSD test. *Thirty-six of the I,242 respondents were excluded for unintelligible open-ended responses $(n=8)$ or tampered with the medication but did not report abuse ( $n=28)$. Non-opioid control data are from NHWS, which do not allow for differentiation of opioid-related costs.

Abbreviations: ER, emergency room; LSD, least significant difference; NHWS, National Health and Wellness Survey. 


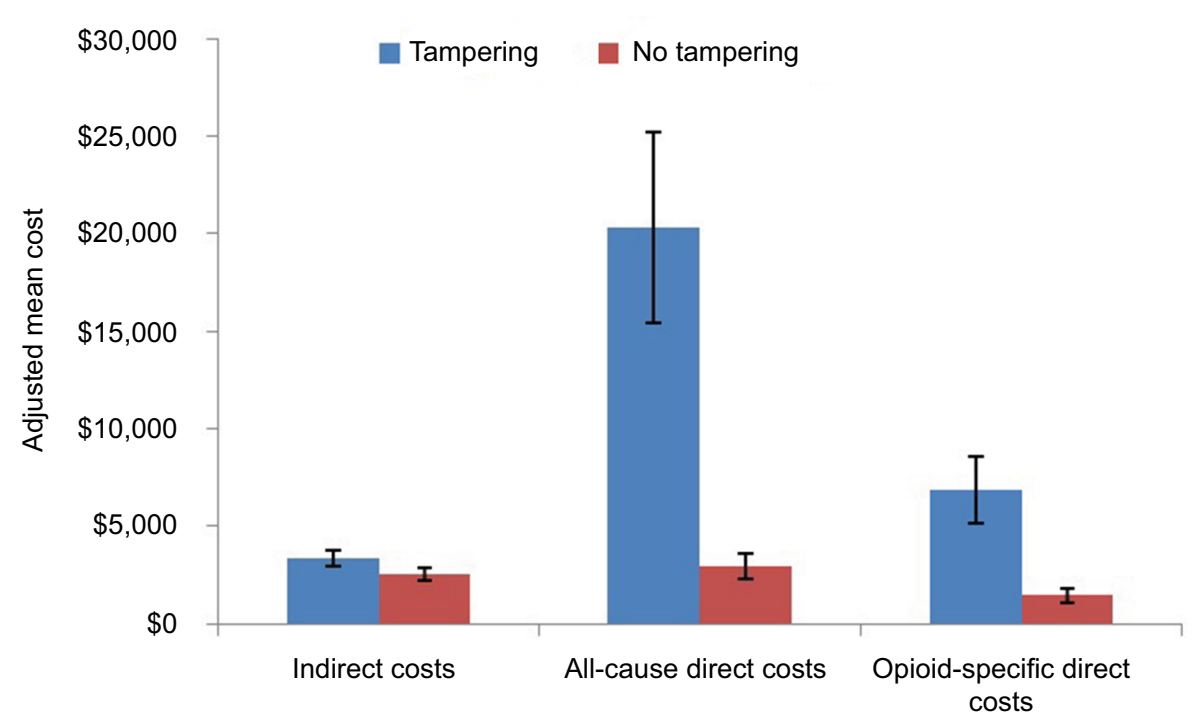

Figure 2 Adjusted 3-month direct and indirect costs for US adults abusing prescription opioids.

Notes: Values are least-squared means from a generalized linear model at the mean of the covariates. Error bars are standard error of the mean. Covariates included in the model were age, sex, annual household income, race, employment status, $\mathrm{CCl}$, self-reported psychiatric illness, obesity, alcohol consumption, smoking, exercise, use of an opioid in the NHWS, and possession of health insurance.

Abbreviations: $\mathrm{CCl}$, Charlson Comorbidity Index; NHWS, National Health and Wellness Survey.

\section{Discussion}

This study surveyed a large sample of US adults with self-reported opioid medication use to provide per-patient estimates of the economic burden of opioid medication abuse with and without tampering. In line with prior work, ${ }^{17}$ individuals who abused opioid medications had substantially increased costs relative to non-abusers, including individuals using opioid medications for their intended medical purposes and control subjects. Moreover, abuse with tampering was associated with substantially higher costs than abuse without tampering. This finding remained consistent after adjusting for relevant covariates.

Chronic opioid abusers who reported tampering with medications were estimated to have the highest costs. Regarding the workforce, those who abused and tampered reported higher mean absenteeism costs, relative to all other groups. Mean costs for work missed (absenteeism) were over $\$ 1,800$ - nearly 3 times higher than those who abused without tampering, more than 3 times higher than those using opioids for their intended medical purpose, and $\sim 10$ times higher than controls. Presenteeism costs were more similar between the two groups who abused and the group using opioids for their intended medical purpose, though it is important to keep in mind that high absenteeism places a limit on presenteeism costs as the time spent impaired at work is limited by the time completely away from work. When total indirect costs were considered, these were highest among those who abused and tampered. Direct costs were higher than indirect costs, and those who abused and tampered incurred an average of over $\$ 23,000$ in estimated direct medical costs for both opioid-related health care and non-opioid-related health care. These costs were over 5 times greater than those incurred by respondents who abused without tampering. Notably, opioidspecific medical costs in those who abused and tampered were nearly quadruple those of respondents who abused, but did not tamper which represented an increase of $\sim \$ 6,500$.

Regression modeling among opioid abusers showed a similar pattern of results, although indirect costs were not significantly higher among those who abused and tampered relative to those who did not tamper. Adjusted all-cause direct costs were more than 6 times as high among those who abused and tampered as those who abused but did not tamper $(>\$ 20,000 \mathrm{vs}$ $\$ 3,000)$. Though respondents attributed most of their medical care to non-opioid-related health problems, costs estimated from opioid-specific medical visits were at nearly $\$ 7,000$ per quarter, almost 5 times as high among those who abused and tampered relative to those who abused but did not tamper.

These data also show a strong connection between tampering and frequency, with those who abused more frequently being more likely to tamper with the medication. The connection between frequency and tampering should not be a surprise as tampering increases the number of ways in which a drug can potentially be abused. Both increased frequency and tampering are likely to be markers of more advanced, 
longer-term abuse or dependence. We chose not to try to disentangle tampering from abuse frequency, but it is likely that some of the differences between the two abusing groups would remain even if tampering was eliminated.

To our knowledge, this is the first study to provide perpatient estimates of the US economic burden of medical opioid abuse according to whether or not the medication was tampered with. These data provide an impetus to focus special efforts on attenuating abuse among those tampering with opioids, and go beyond earlier analysis describing work impairment and health care use. Estimating costs not only allows the magnitude of tampering with prescription opioid medication to be more easily compared with other public health priorities but also allows for impacts of work impairment and disparate types of health care - which cannot otherwise be integrated - to be combined into a single, understandable metric.

\section{Limitations}

There are limitations to the current study inherent to crosssectional, self-reported data. First, opioid use and abuse were self-reported and retrospective and therefore subject to the errors of recall. The results are specific to the definition of abuse adopted for this study, which is narrower here than the NMU often equated with abuse. ${ }^{14}$ Lost work productivity and medical visits were also self-reported, and recall bias may have resulted in over or underestimation. Recall errors may be more pronounced among those who are abusing prescription opioids and frequently accessing medical care. Second, indirect cost estimates were based on average wages, which may not have been representative of the individuals included in the study, and estimates of direct costs were based on average unit costs rather than actual expenses. Third, the data are cross-sectional and, as such, no claims about cause and effect are possible. However, it seems very unlikely that tampering with opioids would be an effect of health care use and work impairment instead of a cause. Finally, it is important to note the lack of data on respondents indicating opioid misuse in the current survey. The screening for the current study required respondents to report taking opioids according to their physician's directions (medical use only group), and/or using the opioid to get high (abuse groups). Respondents to the screener who indicated opioid use and deviation from doctor's orders, but not abuse as defined here, were excluded from the survey.

It is possible that variables aside from tampering would explain some differences in costs between those who do and do not tamper. While some potential confounders were controlled for using regression, there could be other unmeasured variables that account for some of the observed differences in outcomes. As already noted above, those who tampered were overwhelmingly frequent abusers of prescription opioids, and no attempt was made to control for frequency in the regression models. We should also note that analyses were repeated using alternative sources of unit costs, and these confirmed the significant relationship between tampering and direct medical costs (data available from the corresponding author). Notably, the present study did not collect information on abuse of other drugs, but individuals who abuse prescription opioids through tampering may be more likely to abuse other drugs as well. If so, some of the incremental cost of tampering would likely be attributable to abuse of other drugs if non-opioid drug abuse was more common and/or more severe among those who tampered with prescription opioids. This is an important point when considering whether to invest in interventions to combat opioid tampering, whether through education, prescribing other types of analgesics, or abuse-deterrent formulations of opioids. However, the magnitude of the elevated costs among those who abused and tampered with prescription opioids relative to those who abused without tampering argue for continued research to reduce the impact of opioid abuse.

\section{Conclusion}

Ultimately, study results indicate opioid abuse to be associated with significant economic costs, and this is particularly true when individuals tamper with the medication. In light of the findings from this study, measures to prevent or reduce the incidence of opioid tampering may help to reduce the substantial impact of opioid abuse on the workforce and health care systems. Policymakers and practitioners should consider the high cost per tampering individual when evaluating such measures.

\section{Acknowledgments}

This study was sponsored by Pfizer. Editorial/medical writing support was provided by Tammy Schuler and Martine C. Maculaitis at Kantar Health and was funded by Pfizer. These results were presented as a poster at the 2015 International Meeting of the International Society of Pharmacoeconomics and Outcomes Research in Philadelphia, Pennsylvania, in June 2015. The abstract for that presentation was published as: Cost of tampering in abuse of prescription opioids. Value Health. 2015;18(3):A120.

\section{Disclosure}

Jeffrey Vietri was an employee of Kantar Health during the development of this paper, Kantar Health are paid consultants to Pfizer in connection with this study and the development 
of this paper. Jeffrey Vietri, Elizabeth Masters, Alexandra Barsdorf, and Jack Mardekian are employees of and hold stock options for Pfizer. The authors report no other conflicts of interest in this work.

\section{References}

1. Atluri S, Boswell MV, Hansen HC, Trescot AM, Singh V, Jordan AE. Guidelines for the use of controlled substances in the management of chronic pain. Pain Physician. 2003;6(3):233-2578.

2. President Donald J. Trump is Taking Action on Drug Addiction and the Opioid Crisis. Available from: https://www.whitehouse.gov/briefingsstatements/president-donald-j-trump-taking-action-drug-addictionopioid-crisis/. Accessed February 2, 2018.

3. Substance Abuse and Mental Health Services Administration, Center for Behavioral Health Statistics and Quality. Results from the 2013 National Survey on Drug Use and Health: Detailed Tables. Rockville, MD, USA: SAMHSA; 2014.

4. Birnbaum HG, White AG, Schiller M, Waldman T, Cleveland JM, Roland CL. Societal costs of prescription opioid abuse, dependence, and misuse in the United States. Pain Med. 2011;12(4):657-667.

5. Jones CM. Frequency of prescription pain reliever nonmedical use: 2002-2003 and 2009-2010. Arch Intern Med. 2012;172(16):1265-1267.

6. Wide-ranging Online Data for Epidemiologic Research (WONDER), Multiple-Cause-of-Death file, 2000-2014; 2015. Available from: http:// www.cdc.gov/nchs/data/health_policy/AADR_drug_poisoning_involving_OA_Heroin_US_2000-2014.pdf. Accessed July 31, 2017.

7. Centers for Disease Control and Prevention. Prescription drug overdose in the United States: Fact sheet. Available from: http://www.cdc.gov/ drugoverdose/index.html. 2015. Accessed November 20, 2016.

8. Centers for Disease Control and Prevention (CDC). Vital signs: overdoses of prescription opioid pain relievers-United States, 1999-2008. MMWR Morb Mortal Wkly Rep. 2011;60(43):1487-1492.

9. Centers for Disease Control and Prevention (CDC). CDC grand rounds: prescription drug overdoses-a US epidemic. MMWR Morb Mortal Wkly Rep. 2012;61(1):10-13.

10. Centers for Disease Control and Prevention. Number of poisoning deaths involving opioid analgesics and other drugs or substances - United States, 1999-2010. MMWR Morb Mortal Wkly Rep. 2013;62(12):234-235.
11. Volkow ND, McLellan TA. Curtailing diversion and abuse of opioid analgesics without jeopardizing pain treatment. JAMA. 2011;305(13):1346-1347.

12. Substance Abuse and Mental Health Services Adminstration. Drug Abuse Warning Network, 2011: National Estimates of Drug-Related Emergency Department Visits. Rockville, MD, USA: SAMHSA; 2013.

13. Dowell D, Haegerich TM, Chou R. CDC guideline for prescribing opioids for chroinc pain - United States, 2016. JAMA. 2016;315(15): 1624-1645.

14. Katz N, Dart RC, Bailey E, Trudeau J, Osgood E, Paillard F. Tampering with prescription opioids: nature and extent of the problem, health consequences, and solutions. Am J Drug Alcohol Abuse. 2011;37(4):205-217.

15. Vietri J, Joshi AV, Barsdorf AI, Mardekian J. Prescription opioid abuse and tampering in the United States: results of a self report survey. Pain Med. 2014;15(12):2064-2074.

16. Surratt H, Kurtz SP, Cicero TJ. Alternate routes of administration and risk for HIV among prescription opioid abusers. J Addict Dis. 2011;30(4):334-341.

17. Meyer R, Patel AM, Rattana SK, Quock TP, Mody SH. Prescription opioid abuse: a literature review of the clinical and economic burden in the United States. Popul Health Manag. 2014;17(6):372-387.

18. Chandwani HS, Strassels SA, Rascati KL, Lawson KA, Wilson JP. Estimates of charges associated with emergency department and hospital inpatient care for opioid abuse-related events. J Pain Palliat Care Pharmacother. 2013;27(3):206-213.

19. Baser O, Xie L, Mardekian J, SchaafD, Wang L, Joshi AV. Prevalence of diagnosed opioid abuse and its economic burden in the veterans health administration. Pain Pract. 2014;14(5):437-445.

20. Charlson ME, Pompei P, Ales KL, MacKenzie CR. A new method of classifying prognostic comorbidity in longitudinal studies: development and validation. J Chron Dis. 1987;40(5):373-383.

21. Reilly MC, Zbrozek AS, Dukes EM. The validity and reproducibility of a work productivity and activity impairment instrument. Pharmacoeconomics. 1993;4(5):353-365.

22. Bureau of Labor Statistics. Highlights of women's earnings in 2011. Report 1038. Available from: http://www.bls.gov/cps/cpswom 2011. pdf. 2012. Accessed November 20, 2016.

23. Substance Abuse and Mental Health Services Administration. The ADSS Cost Study: Costs of Substance Abuse Treatment in the Specialty Sector. Rockville, MD, USA: Office of Applied Studies (DHHS Publication No. SMA 03-3765, Analytic Series A-20); 2003.
ClinicoEconomics and Outcomes Research

\section{Publish your work in this journal}

ClinicoEconomics and Outcomes Research is an international, peerreviewed open-access journal focusing on health technology assessment, pharmacoeconomics and outcomes research in the areas of diagnosis, medical devices, and clinical, surgical and pharmacological intervention. The economic impact of health policy and health systems
Submit your manuscript here: https://www.dovepress.com/clinicoeconomics-and-outcomes-research-journal organization also constitute important areas of coverage. The manuscript management system is completely online and includes a very quick and fair peer-review system, which is all easy to use. Visit http://www.dovepress.com/testimonials.php to read real quotes from published authors. 